\title{
Dynamo action and magnetic buoyancy in convection simulations with vertical shear
}

\author{
G. Guerrero ${ }^{1}$ and P. J. Käpylä ${ }^{1,2}$ \\ 1 NORDITA, Roslagstullsbacken 23, 10691 Stockholm, Sweden \\ e-mail: guerrero@nordita.org \\ 2 Department of Physics, Gustaf Hällströmin katu 2a, PO Box 64, 00014 University of Helsinki, Finland
}

Received 18 February 2011 / Accepted 11 July 2011

ABSTRACT

\begin{abstract}
Context. A hypothesis for sunspot formation is the buoyant emergence of magnetic flux tubes created by the strong radial shear at the tachocline. In this scenario, the magnetic field has to exceed a threshold value before it becomes buoyant and emerges through the whole convection zone.

Aims. We follow the evolution of a random seed magnetic field with the aim of study under what conditions it is possible to excite the dynamo instability and whether the dynamo generated magnetic field becomes buoyantly unstable and emerges to the surface as expected in the flux-tube context.

Methods. We perform numerical simulations of compressible turbulent convection that include a vertical shear layer. Like the solar tachocline, the shear is located at the interface between convective and stable layers.

Results. We find that shear and convection are able to amplify the initial magnetic field and form large-scale elongated magnetic structures. The magnetic field strength depends on several parameters such as the shear amplitude, the thickness and location of the shear layer, and the magnetic Reynolds number (Rm). Models with deeper and thicker tachoclines allow longer storage and are more favorable for generating a mean magnetic field. Models with higher Rm grow faster but saturate at slightly lower levels. Whenever the toroidal magnetic field reaches amplitudes greater a threshold value which is close to the equipartition value, it becomes buoyant and rises into the convection zone where it expands and forms mushroom shape structures. Some events of emergence, i.e. those with the largest amplitudes of the initial field, are able to reach the very uppermost layers of the domain. These episodes are able to modify the convective pattern forming either broader convection cells or convective eddies elongated in the direction of the field. However, in none of these events the field preserves its initial structure. The back-reaction of the magnetic field on the fluid is also observed in lower values of the turbulent velocity and in perturbations of approximately three per cent on the shear profile.

Conclusions. The results indicate that buoyancy is a common phenomena when the magnetic field is amplified through dynamo action in a narrow layer. It is, however, very hard for the field to rise up to the surface without losing its initial coherence.
\end{abstract}

Key words. magnetohydrodynamics (MHD) - convection - turbulence - Sun: dynamo - stars: magnetic field

\section{Introduction}

Sunspots appear at the solar surface following an 11-year cycle. They reveal the presence of strong magnetic fields in the solar interior, and suggest the existence of a dynamo process governing its evolution. However, the process by which sunspots are formed is still debated. At the solar surface, sunspots are observed as bipolar patches of radial magnetic field. This intuitively suggests that they are formed by the emergence of horizontal concentrations of magnetic field lines, often called magnetic flux tubes. Since Parker's original proposal of magnetic buoyancy (Parker 1955), buoyancy models have evolved through the thin flux tube approximation (Spruit 1981; Caligari et al. 1995; Fan \& Fisher 1996), to numerical simulations of the emergence of 3D magnetic flux tubes. These have studied the rise of magnetic flux tubes either from the base of the convection zone (e.g. Fan 2001; Abbett et al. 2001; Fan 2008) or from the uppermost subsurface layers (see e.g., Cheung et al. 2008; Martínez-Sykora et al. 2008, and references therein). The buoyant rise of flux tubes from the tachocline up to the surface is currently the most widely accepted mechanism of sunspot formation. During the last three decades much work has been done in order to understand the buoyancy phenomena and to reconcile the results of flux tube models with phenomenological sunspots rules such as Joy's law or the topological difference between the two spots in a pair. In spite of the fact that these basic observations have been qualitatively reproduced by the flux tube models, a set of complications has questioned the feasibility of this scenario. These may be summarized as follows:

Magnetic flux tubes at the base of the convection zone should have a strength of around $10^{5} \mathrm{G}$ in order to become unstable and then cross the entire convection zone up to the surface (Caligari et al. 1995). This has been considered a problem since the magnetic energy density corresponding to such a field is one to two orders of magnitude larger than the kinetic energy of the turbulent motions (the equipartition energy). Note, however, that in the presence of shear this is not any longer an upper limit for the amplitude of the magnetic field (see e.g. Käpylä \& Brandenburg 2009).

Sunspots are coherent magnetic structures which means that the flux tubes should preserve their integrity while they rise through the entire convection zone. However, this region is highly turbulent and stratified, spanning more than 20 pressure scale heights, so in addition to the $10^{5} \mathrm{G}$ strength, the tubes must have a certain amount of twist in order to resist the turbulent diffusion (see e.g. Emonet \& Moreno-Insertis 1998; Fan et al. 2003). The current results do not conclusively yield the amount of twist required for the tubes to rise coherently up to 
the surface. On one hand, 2D simulations require a larger twist in order to prevent the fragmentation of the flux tube in two tubes due to the vorticity generated by the buoyant rise (Schüssler 1979; Moreno-Insertis \& Emonet 1996; Longcope et al. 1996; Emonet \& Moreno-Insertis 1998; Fan et al. 1998). On the other hand, rising 3D flux tubes require less twist thanks to the tension forces due to the longitudinal curvature of the tube (Fan 2001). Three dimensional simulations in spherical coordinates require fine tuning of the initial twist for the tubes to emerge to the surface with the observed tilt (Fan 2008).

Magnetohydrodynamic simulations of convection in Cartesian coordinates have been able to produce large-scale magnetic fields through $\alpha^{2}$ (Käpylä et al. 2009b) and $\alpha \Omega$ (Käpylä et al. 2008) dynamo action. However, the magnetic fields generated in these simulations are more homogeneously distributed in space rather than in the form of isolated magnetic structures. To date, however, no direct simulations, starting from the amplification of a seed magnetic field, have been able to spontaneously form sunspot-like magnetic structures.

Furthermore, instead of showing signs of buoyant rise and emergence of the magnetic field, the 3D dynamo simulations above have shown that the magnetic field is pumped down by convective downflows and tends to remain in the stable layer (see also Tobias et al. 1998, 2001; Ossendrijver et al. 2002). Fan et al. (2003) studied the evolution of an isolated twisted flux tube in a turbulent convection zone. They found that coherent rise is possible as far as the magnetic buoyant force overcomes the hydrodynamic force from convection, i.e., obeying the condition $B_{0}>\left(H_{\mathrm{P}} / a\right)^{1 / 2} B_{\text {eq }}$, where $B_{0}$ is the initial magnetic field strength, $H_{\mathrm{P}}$ is the pressure scale height, and $a$ is the radius of the tube. In their case where $\left(H_{\mathrm{P}} / a\right)^{1 / 2} \approx 3$, magnetic flux tubes with $B_{0} \geq 3 B_{\text {eq }}$ are able to rise coherently without being affected by convection. Similar results were found in a similar setup in spherical geometry (Jouve \& Brun 2009). However, this does not address the issue of pumping since, firstly, a strong magnetic flux tube is imposed in the convective layer, and secondly, magnetic pumping is an effect related to the gradient of turbulence intensity (Kichatinov \& Rüdiger 1992), which is not important in these simulations.

In addition to the thin flux tube approximation and simulations of rising flux tubes in stratified atmospheres, other attempts have found the formation of magnetic structures due to localized shear layers (Cline et al. 2003). They found that if the initial magnetic field is weak, its evolution is described be diffusive decay. On the other hand, if the imposed field is sufficiently large to become buoyant, then a hydrodynamic, of Kelvin-Helmholtz type, instability, together with the buoyancy instability will produce an $\alpha$ effect able to sustain the dynamo. Convection is not considered in this case. More recent efforts have been made in order to simulate the formation of a magnetic layer through the interaction of an imposed shear in convectively stable (Vasil \& Brummell 2008) and unstable (Silvers et al. 2009a) atmospheres, with an imposed vertical magnetic field. They have found that, unlike in the cases of imposed toroidal magnetic layers, the buoyancy instability is harder to excite when the magnetic field is generated by the shear. More recently Silvers et al. (2009b), have found, with a similar setup, that the buoyancy may be favored by the presence of double-diffusive instabilities (these in turn depend on the ratio between the thermal and magnetic diffusivities, $\chi / \eta$, often known as the inverse Roberts number). Later independent study of Chatterjee et al. (2011) has confirmed this result. However, in most of the current models, the presence of stratified turbulence, self-consistent generation of the magnetic field, or both, are omitted.
In view of the above mentioned issues, other mechanisms have been proposed in order to explain sunspots. These are related to instabilities due to the presence of a diffuse largescale magnetic field in a highly stratified turbulent medium (Kleeorin \& Rogachevskii 1994; Rogachevskii \& Kleeorin 2007; Brandenburg et al. 2010a,b; Käpylä et al. 2011). Meanfield models using this mechanism are able to produce strong flux concentrations, but this has not yet been achieved in direct numerical simulations.

Motivated by the lack of simulations that merge in one model the several processes that so far have been simulated separately, here we present numerical simulations of compressible turbulent convection with an imposed flow with radial shear located in a sub-adiabatic layer beneath the convective region. For numerical reasons we do not include rotation in our setup, i.e. the turbulence is not helical, and an $\alpha \Omega$ dynamo is not expected. Nevertheless, recent studies have shown that mean-field dynamo action is possible due to non-helical turbulence and shear (Brandenburg 2005a; Yousef et al. 2008a,b; Brandenburg et al. 2008). The nature of this dynamo is not yet entirely clear and may be attributed to the so called shear-current effect (Rogachevskii \& Kleeorin 2003, 2004) or to the incoherent (stochastic) $\alpha$-effect (Vishniac \& Brandenburg 1997). According to Brandenburg et al. (2008) the latter explanation is consistent with the turbulent transport coefficients.

Based on these results, we expect the development of a mean magnetic field, i.e. dynamo action, in a system that mimics, as far as possible, the conditions of the solar interior, specifically in the lower part of the convection zone and the tachocline. As the shear is localized in a very narrow layer, we also expect the formation of a magnetic layer and the subsequent buoyant rise of magnetic field structures.

A similar setup was studied recently by Tobias et al. (2008). They reported the appearance of elongated stripes of magnetic field in the direction of the shear. However, since they used the Boussinesq approximation in their simulations, no magnetic buoyancy could occur. They also do not report the presence of a large scale dynamo.

Two important features distinguish the simulations presented here from previous studies in the context of flux tube formation and emergence. Firstly, we consider a highly stratified domain with $\approx 8$ scale heights in pressure and $\approx 6$ scale heights in density. Secondly, we do not impose a radial magnetic background field but allow the self-consistent development of the field from a initial random seed.

Even though this is a complicated setup where it is difficult to analyze the different processes independently, we believe that these simulations may shed some light on the current paradigm of sunspot formation. There are several important issues that we want to address with the following simulations. (1) What are the requirements for dynamo action in the present setup? This includes the dependence of the dynamo excitation on several parameters such as the amplitude of the shear, thickness and location of the shear layer, and the aspect ratio of the box. (2) What is the resulting configuration of the magnetic field? In particular, is the field predominantly in small or large scales, and is it organized in the form of a magnetic layer or isolated magnetic flux tubes? (3) Is the buoyancy instability (Parker 1955) operating on these magnetic structures? If yes, (4) how does it depend on the parameters listed above? (5) Is it possible to have magnetic structures strong enough to emerge from the shear layer to the surface without being affected by the turbulent convective motions? (6) Finally, it is important to study how these strong 
structures react back on the fluid motions, including the shear profile as well as the convective pattern.

Another important issue that may be addressed in this context is the mechanism that triggers the dynamo instability. With the recent developments on the test-field method, it is possibly to compute the dynamo transport coefficients and have a better understanding on the underlying mechanism.

We have organized this paper as follow: in Sect. 2 we provide the details of the numerical model, in Sect. 3 we describe our results. We summarize and conclude in Sect. 4.

\section{The model}

Our model setup is similar to that used by e.g. Brandenburg et al. (1996) and Käpylä et al. (2008). A rectangular portion of a star is modeled by a box whose dimensions are $\left(L_{x}, L_{y}, L_{z}\right)=(4,4,2) d$, where $d$ is the depth of the convectively unstable layer, which is also used as the unit of length. The box is divided into three layers, an upper cooling layer, a convectively unstable layer, and a stable overshoot layer (see below). The following set of equations for compressible magnetohydrodynamics is being solved:

$$
\frac{\partial \boldsymbol{A}}{\partial t}=\boldsymbol{U} \times \boldsymbol{B}-\mu_{0} \eta \boldsymbol{J}
$$

$\frac{D \ln \rho}{D t}=-\boldsymbol{\nabla} \cdot \boldsymbol{U}$

$\frac{D \boldsymbol{U}}{D t}=-\frac{1}{\rho} \boldsymbol{\nabla} p+\boldsymbol{g}+\frac{1}{\rho} \boldsymbol{J} \times \boldsymbol{B}+\frac{1}{\rho} \boldsymbol{\nabla} \cdot 2 v \rho \mathbf{S}-\frac{\boldsymbol{U}-\overline{\boldsymbol{U}}^{(0)}}{\tau_{\mathrm{f}}}$,

$T \frac{D s}{D t}=\frac{1}{\rho} \boldsymbol{\nabla} \cdot K \boldsymbol{\nabla} T+2 v \mathbf{S}^{2}+\frac{\mu_{0} \eta}{\rho} J^{2}-\Gamma_{\text {cool }}$,

where $D / D t=\partial / \partial t+\boldsymbol{U} \cdot \boldsymbol{\nabla}$ is the total time derivative. $\boldsymbol{A}$ is the magnetic vector potential, $\boldsymbol{B}=\boldsymbol{\nabla} \times \boldsymbol{A}$ is the magnetic field, and $\boldsymbol{J}=\boldsymbol{\nabla} \times \boldsymbol{B} / \mu_{0}$ is the current density, $\mu_{0}$ is the magnetic permeability, $\eta$ and $v$ are the magnetic diffusivity and kinematic viscosity, respectively, $T$ is the temperature, $s$ is the specific entropy, $K$ is the heat conductivity, $\rho$ is the density, $\boldsymbol{U}$ is the velocity, and $\boldsymbol{g}=-g \hat{z}$ is the gravitational acceleration. The fluid obeys an ideal gas law $p=\rho e(\gamma-1)$, where $p$ and $e$ are the pressure and internal energy, respectively, and $\gamma=c_{\mathrm{P}} / c_{\mathrm{V}}=5 / 3$ is the ratio of specific heats at constant pressure and volume, respectively. The specific internal energy per unit mass is related to the temperature via $e=c_{\mathrm{V}} T$. The rate of strain tensor $\mathbf{S}$ is given by

$\mathrm{S}_{i j}=\frac{1}{2}\left(U_{i, j}+U_{j, i}\right)-\frac{1}{3} \delta_{i j} \boldsymbol{\nabla} \cdot \boldsymbol{U}$,

where the commas denote derivatives. The last term of Eq. (3) relaxes the velocity $\boldsymbol{U}$ towards a target profile $\overline{\boldsymbol{U}}^{(0)}$ where $\tau_{\mathrm{f}}=$ $2 \sqrt{(} d / g)$ is a relaxation time scale. In a non-shearing case this value corresponds to $\approx 1 / 2 \tau_{\text {turn }}$, where $\tau_{\text {turn }}=\left(u_{\text {rms }} k_{\mathrm{f}}\right)^{-1}$ gives an estimate of the convective turnover time. The target profile is chosen so that it mimics the radial shear in the solar tachocline. More details of the shear profiles are given in Sect. 2.3.

The last term of Eq. (4) describes cooling at the top of the domain according to

$\Gamma_{\text {cool }}=\Gamma_{0} f(z)\left(\frac{T-T_{4}}{T_{4}}\right)$,

(6) $\mathrm{Sh}=\frac{U_{0} / d_{\mathrm{s}}}{u_{\mathrm{rms}} k_{\mathrm{f}}}$, where $f(z)$ is a profile function equal to unity in $z>z_{3}$ and smoothly going to to zero below, and $\Gamma_{0}$ is a specific cooling luminosity chosen so that the temperature in the uppermost layer relaxes toward $T_{4}=T\left(z=z_{4}\right)$.

The positions of the bottom of the box, bottom and top of the convectively unstable layer, and the top of the box, respectively, are given by $\left(z_{1}, z_{2}, z_{3}, z_{4}\right)=(-0.8,0,1,1.2) d$. Initially the stratification is piecewise polytropic with polytropic indices $\left(m_{1}, m_{2}, m_{3}\right)=(6,1,1)$, which leads to a convectively unstable layer above a stable layer at the bottom of the domain and an isothermal cooling layer at the top.

\subsection{Nondimensional units and parameters}

Dimensionless quantities are obtained by setting

$d=g=\rho_{0}=c_{\mathrm{P}}=\mu_{0}=1$,

where $\rho_{0}$ is the initial density at $z_{2}$. The units of length, time, velocity, density, entropy, and magnetic field are

$[x]=d, \quad[t]=\sqrt{d / g}, \quad[U]=\sqrt{d g}, \quad[\rho]=\rho_{0}$,
$[s]=c_{\mathrm{P}}, \quad[B]=\sqrt{d g \rho_{0} \mu_{0}}$.

We define the fluid and magnetic Prandtl numbers and the Rayleigh number as

$\operatorname{Pr}=\frac{v}{\chi_{0}}, \quad \operatorname{Pm}=\frac{v}{\eta}, \quad \mathrm{Ra}=\frac{g d^{4}}{v \chi_{0}}\left(-\frac{1}{c_{\mathrm{P}}} \frac{\mathrm{d} s}{\mathrm{~d} z}\right)_{0}$,

where $\chi_{0}=K /\left(\rho_{\mathrm{m}} c_{\mathrm{P}}\right)$ is the thermal diffusivity, and $\rho_{\mathrm{m}}$ is the density in the middle of the unstable layer. For the magnetic diffusivity we consider a $z$-dependent profile which gives an order of magnitude smaller value in the radiative layer than in the convection zone through a smooth transition. Thus the magnetic Prandtl number in the radiative layer is $\mathrm{Pm}=15$ and in the convection zone $\mathrm{Pm}=1.5$.

The entropy gradient, measured in the middle of the convectively unstable layer in the initial non-convecting hydrostatic state, is given by

$\left(-\frac{1}{c_{\mathrm{P}}} \frac{\mathrm{d} s}{\mathrm{~d} z}\right)_{0}=\frac{\nabla-\nabla_{\mathrm{ad}}}{H_{\mathrm{P}}}$

where $\nabla-\nabla_{\mathrm{ad}}$ is the superadiabatic temperature gradient with $\nabla_{\mathrm{ad}}=1-1 / \gamma, \nabla=(\partial \ln T / \partial \ln p)_{z_{\mathrm{m}}}$, and where $z_{\mathrm{m}}=\frac{1}{2}\left(z_{3}+\right.$ $\left.z_{2}\right)$. The amount of stratification is determined by the parameter $\xi_{0}=(\gamma-1) c_{\mathrm{V}} T_{4} /(g d)$, which is the pressure scale height at the top of the domain normalized by the depth of the unstable layer. We use in all cases $\xi_{0}=0.12$, which results in a density contrast of about 120 . We define the fluid and magnetic Reynolds numbers via

$\operatorname{Re}=\frac{u_{\mathrm{rms}}}{v k_{\mathrm{f}}}, \quad \mathrm{Rm}=\frac{u_{\mathrm{rms}}}{\eta k_{\mathrm{f}}}=\operatorname{PmRe}$,

where $u_{\mathrm{rms}}$ is the rms value of the velocity fluctuations and $k_{\mathrm{f}}=$ $2 \pi / d$ is assumed as a reasonable estimate for the wavenumber of the energy-carrying eddies. Note that due to our definitions of the Reynolds numbers they are smaller than the usually adopted ones by a factor of $2 \pi$. The amount of shear is quantified by 
Table 1. Summary of the runs.

\begin{tabular}{lcccccccccccc}
\hline \hline Run & $U_{0} /(d g)^{1 / 2}$ & $d_{z}$ & $z_{\text {ref }}$ & $\mathrm{Sh}$ & $\mathrm{Re}$ & $\mathrm{Rm}$ & $\mathrm{Ma}$ & $\lambda\left[10^{-2}\right]$ & $B_{\mathrm{eq}}$ & $\tilde{B}_{\text {rms }}$ & $\tilde{B}_{y}$ & $\bar{B} / \bar{B}_{\mathrm{T}}$ \\
\hline S00 & 0.00 & 0.05 & -0.05 & 0.0 & 8.9 & 14.7 & 0.028 & -2.16 & 0.06 & - & - & - \\
S01 & 0.06 & 0.05 & -0.05 & 3.7 & 8.2 & 13.6 & 0.026 & -1.29 & 0.05 & - & - & - \\
S02 & 0.09 & 0.05 & -0.05 & 6.8 & 13.4 & 22.3 & 0.042 & -0.34 & 0.21 & - & - & - \\
S03 & 0.12 & 0.05 & -0.05 & 7.3 & 15.3 & 25.5 & 0.053 & 0.03 & 0.24 & - & - & - \\
S04 & 0.15 & 0.05 & -0.05 & 7.6 & 19.9 & 33.2 & 0.062 & 1.72 & 0.40 & 0.31 & 5.32 & 0.34 \\
\hline D01 & 0.15 & 0.05 & -0.05 & 7.6 & 19.9 & 33.2 & 0.062 & 1.72 & 0.40 & 0.31 & 5.32 & 0.34 \\
D02 & 0.15 & 0.05 & -0.09 & 7.7 & 19.7 & 32.8 & 0.062 & 1.72 & 0.39 & 0.40 & 4.92 & 0.36 \\
D03 & 0.15 & 0.05 & -0.13 & 7.6 & 19.9 & 33.1 & 0.062 & 1.36 & 0.39 & 0.42 & 4.83 & 0.36 \\
D04 & 0.15 & 0.05 & -0.17 & 7.3 & 20.1 & 34.9 & 0.066 & 1.37 & 0.39 & 0.56 & 6.15 & 0.37 \\
\hline T01 & 0.15 & 0.05 & -0.05 & 7.6 & 19.9 & 33.2 & 0.062 & 1.72 & 0.40 & 0.31 & 5.32 & 0.34 \\
T02 & 0.15 & 0.07 & -0.05 & 6.0 & 18.1 & 30.1 & 0.057 & 1.00 & 0.33 & 0.34 & 4.79 & 0.36 \\
T03 & 0.15 & 0.09 & -0.05 & 4.9 & 17.2 & 28.7 & 0.054 & 0.91 & 0.30 & 0.31 & 5.40 & 0.37 \\
T04 & 0.15 & 0.11 & -0.05 & 4.4 & 15.8 & 26.3 & 0.049 & 0.88 & 0.26 & 0.44 & 6.15 & 0.41 \\
T04 & 0.15 & 0.11 & -0.05 & 3.8 & 18.1 & 30.2 & 0.056 & 1.18 & 0.36 & 0.40 & 3.75 & 0.39 \\
T05 & 0.15 & 0.15 & -0.05 & 3.4 & 15.0 & 24.9 & 0.045 & 0.39 & 0.24 & - & - & - \\
T06 & 0.15 & 0.20 & -0.05 & 2.9 & 13.1 & 22.0 & 0.041 & -0.04 & 0.19 & - & - & - \\
\hline
\end{tabular}

Notes. Here Ma $=u_{\mathrm{rms}} /(g d)^{1 / 2}, \lambda$ is the growth rate in units of $\tau_{\mathrm{turn}}, \tilde{B}_{\mathrm{rms}} \equiv B_{\mathrm{rms}} / B_{\text {eq }}, \tilde{B}_{y} \equiv \max \left(\left|B_{y}\right|\right) / B_{\text {eq }}$, where the equipartition magnetic field is evaluated at $z=z_{\text {ref }}$, see Eq. (13), and is given in units of $\sqrt{d g \rho_{0} \mu_{0}}$. Finally $\bar{B} / \bar{B}_{\mathrm{T}}=\sqrt{\langle\boldsymbol{B}\rangle_{y}^{2} /\left\langle\boldsymbol{B}_{y}^{2}\right\rangle}$ corresponds to fraction of mean magnetic field compared with the total field. The numbers are given for the saturated state of the dynamo. In all these simulations the resolution is $128^{3}$ grid points, $\operatorname{Pr}=20, \operatorname{Pm}=15$ in the stable layer and 1.5 in the convection zone, and $\operatorname{Ra} \simeq 8.3 \times 10^{6}$.

where $U_{0}$ is the amplitude and $d_{\mathrm{s}}$ the half-width of the imposed shear profile (see below). The equipartition magnetic field is defined by

$$
B_{\text {eq }} \equiv\left(\mu_{0} \overline{\rho \boldsymbol{U}^{2}}\right)_{z_{\text {ref }}}^{1 / 2} \text {. }
$$

For a better comparison between the magnetic and kinetic energies, we evaluate $B_{\text {eq }}$ at the center of the shear layer, $z=z_{\text {ref }}$.

The simulations were performed with the PencIL Code ${ }^{1}$, which uses sixth-order explicit finite differences in space and third order accurate time stepping method.

\subsection{Boundary conditions}

In the horizontal ( $x$ and $y$ ) directions we use periodic boundary conditions and at the vertical $(z)$ boundaries we use stress-free, impenetrable, boundary conditions for the velocity,

$U_{x, z}=U_{y, z}=U_{z}=0$.

For the magnetic field the vertical field condition is used on the upper boundary whereas the perfect conductor condition is used at the lower boundary, i.e.

$B_{x}=B_{y}=0, \quad\left(z=z_{4}\right)$

$B_{x, z}=B_{y, z}=B_{z}=0, \quad\left(z=z_{1}\right)$

respectively. The upper boundary thus allows magnetic helicity flux out of the box whereas the lower boundary does not. This is likely to be representative of the situation in a real star where magnetic helicity can escape via the surface but does not penetrate into the core.

\subsection{Shear at the base of the convection zone}

In order to mimic the tachocline at the base of the solar convection zone we introduce a shear profile

$\overline{\boldsymbol{U}}^{(0)}=\frac{1}{2} U_{0} \tanh \left(\frac{z-z_{\mathrm{ref}}}{d_{\mathrm{s}}}\right) \hat{\boldsymbol{e}}_{y}$,

\footnotetext{
${ }^{1}$ http://pencil-code.googlecode.com/
}

where $z_{\text {ref }}$ is the reference position of the shear layer. Given the uncertainties of the radial position and width of the tachocline we perform a parameter study where $z_{\text {ref }}$ and $d_{\mathrm{s}}$ are varied. Furthermore, it is of general interest to study how dynamo excitation depends on varying $U_{0}$ and the ratio of $U_{0} / d_{\mathrm{s}}$.

In a steady state the relaxation term that is used to impose the flow balances with the viscous term. However, if rotation is added, e.g. with $\boldsymbol{\Omega}=\Omega_{0}(0,0,1)$, the Coriolis force contributes to the $x$-component of the velocity. In order to balance this term, e.g. with viscous term, an additional flow $U_{x}(z)$ has to be generated. Such $U_{x}(z)$ will then backreact on $U_{y}$ and the resulting shear will be very different than that we wish to impose. Thus we refrain from adding rotation in the model in the present study.

\section{Results}

With the purpose of addressing the questions raised in the introduction, we perform a series of simulations with the model described above where the properties of the shear layer are varied. We first study the hydrodynamic properties of the system and the conditions for dynamo excitation. The results of this parameter study are summarized in Table 1 . Then we study the topological and buoyant properties of the magnetic fields generated in some characteristic runs and compare them with models with different aspect ratio and higher resolution (see Table 2). We finalize this section with the study of the magnetic feedback on the plasma motion and the computation of the turbulent coefficients that govern the evolution of mean magnetic fields in our simulations.

\subsection{Hydrodynamic instabilities}

In our simulation setup, two kinds of hydrodynamical instabilities may develop, namely the Kelvin-Helmholtz $(\mathrm{KH})$ instability due to the imposed shear and stratification, and the convective instability due to the superadiabatic stratification in the middle layer. From hydrodynamical runs, we find that the convective instability develops early, at $t \approx 15 \tau_{\text {turn }}\left(\approx 0.8 \tau_{\text {visc }}\right.$, 
G. Guerrero and P. J. Käpylä: Dynamo action due to localized shear below a convection zone

Table 2. Summary of runs with different aspect ratio (upper two rows) and higher resolution (lower rows).

\begin{tabular}{|c|c|c|c|c|c|c|c|c|c|c|c|c|c|c|c|}
\hline Run & $\left(L_{x}, L_{y}, L_{z}\right)$ & $U_{0} /(d g)^{1 / 2}$ & $d_{z}$ & $z_{\text {ref }}$ & $\operatorname{Pr}$ & $\mathrm{Sh}$ & $\mathrm{Re}$ & $\mathrm{Rm}$ & $\mathrm{Ma}_{\mathrm{k}}$ & $\mathrm{Ma}_{\mathrm{s}}$ & $\lambda\left[10^{-2}\right]$ & $B_{\mathrm{eq}}$ & $\tilde{B}_{\mathrm{rms}}$ & $\tilde{B}_{y}$ & $\bar{B} / \bar{B}_{\mathrm{T}}$ \\
\hline AR01 & $(8,4,2) d$ & 0.15 & 0.11 & -0.05 & 20 & 4.4 & 15.8 & 26.3 & 0.050 & 0.050 & 0.89 & 0.25 & 0.50 & 6.08 & 0.40 \\
\hline AR02 & $(4,8,2) \mathrm{d}$ & 0.15 & 0.11 & -0.05 & 20 & 3.8 & 18.4 & 30.6 & 0.058 & 0.058 & 0.30 & 0.32 & 0.22 & 4.73 & 0.29 \\
\hline$\overline{\mathrm{D} 03 b}$ & $(4,4,2) d$ & 0.15 & 0.05 & -0.10 & 12 & 7.9 & 31.8 & 47.7 & 0.062 & 0.060 & 2.57 & 0.43 & 0.31 & 4.16 & 0.31 \\
\hline T04a & $(4,4,2) d$ & 0.15 & 0.1 & -0.05 & 20 & 5.1 & 25.0 & 37.6 & 0.047 & 0.047 & 2.23 & 0.26 & 0.41 & 5.06 & 0.36 \\
\hline T04b & $(4,4,2) \mathrm{d}$ & 0.15 & 0.1 & -0.05 & 12 & 4.9 & 25.6 & 38.5 & 0.052 & 0.048 & 2.19 & 0.27 & 0.43 & 4.80 & 0.37 \\
\hline T04c & $(4,4,2) \mathrm{d}$ & 0.15 & 0.1 & -0.05 & 3 & 4.4 & 28.6 & 43.0 & 0.056 & 0.054 & 1.21 & 0.30 & 0.35 & 4.13 & 0.36 \\
\hline
\end{tabular}

Notes. Most of the quantities here are defined in Table 1. We have added here the scales of the domain, the Prandtl number and the Mach number at the kinematic $\left(\mathrm{Ma}_{\mathrm{k}}\right)$ and saturated $\left(\mathrm{Ma}_{\mathrm{s}}\right)$ stages. Models with different aspect ratio have the same spatial resolution than models in Table 1. In Runs D03b and T04a-c the resolution is $256^{3}$ grid points.

where $\left.\tau_{\text {visc }}=\left(k_{\mathrm{f}}^{2} v\right)^{-1}\right)$, whereas the KH-instability starts to develop at $t \approx 40 \tau_{\text {turn }}\left(\approx 2 \tau_{\text {visc }}\right)$ in runs with the strongest shear. After a few hundred time units the velocity reaches a statistically steady state (constant rms-velocity), and the toroidal velocity achieves the desired shear profile. A thermally relaxed state (constant thermal energy), however, is reached only after a few thousand time units. This time depends on the radiative conductivity $(K)$, but also on the imposed shear, which produces viscous heating that modifies the thermal stratification of the system as it may be seen in the top panels of Fig. 1. The final velocity profile which includes shear, convection, and the KH instability, does not allow the possibility of including rotation in the model. If it is done, the system develops mean field motions in the horizontal direction which are undesirable in the present study, as explained in Sect. 2.3.

\subsection{Dynamo excitation}

In order to study the dynamo excitation we follow the evolution of an initial random seed magnetic field of the order of $10^{-5} B_{\text {eq }}$. Since there is no rotation and because the vertical component of the large-scale vorticity $\bar{W}_{i}=\epsilon_{i j k} \bar{U}_{j, k} \approx 0$, the average kinetic helicity and mean-field $\alpha$-effect are expected to vanish (e.g. Krause \& Rädler 1980). However, it is still possible to excite a large-scale dynamo due to non-helical turbulence and shear (Brandenburg 2005a; Yousef et al. 2008a,b; Brandenburg et al. 2008). In this part of our study we vary three parameters: the shear amplitude, $U_{0}$ (Runs S00-S04), the location of the shear layer in the convectively stable region, $z_{\text {ref }}$ (Runs D02-D04), and the thickness of the shear region, $2 d_{\mathrm{S}}$ (Runs T02-T04).

We note that no small-scale dynamo is excited in the nonshearing case. With the runs in Set $\mathrm{S}$ we find that the critical value of shear above which a dynamo is excited is $\mathrm{Sh} \approx 7$ (see the top panel of Fig. 2). The value of Sh is roughly three times larger than that computed with typical values of the rmsvelocity and length scales in the solar tachocline ${ }^{2}$.

In Set $\mathrm{D}$, we move the shear layer deeper down into the stable layer, where the turbulent diffusivity is expected to be less, thus allowing a longer storage of the magnetic field ${ }^{3}$. From the hydrodynamical point of view, these simulations have a convection zone that, due to viscous heating, extends into the initially stable layer in the saturated state as it can be seen in the vertical profile of entropy corresponding to Run D04 (red line) in Fig. 1. The three bottom panels of the same figure (see also

\footnotetext{
2 We have considered $\Delta \Omega=33 \mathrm{nHz}, r_{\mathrm{tac}}=0.7 R_{\odot}, d_{\mathrm{tac}}=0.05 R_{\odot}($ e.g. Christensen-Dalsgaard \& Thompson 2007, and references therein), $u_{\mathrm{rms}}=10^{4} \mathrm{~cm} \mathrm{~s}^{-1}$, and $k_{\mathrm{f}}=2 \pi / l$, with $l=10^{10} \mathrm{~cm}$ (see Table 1 of Brandenburg \& Subramanian 2005), which result in $\mathrm{Sh}_{\mathrm{tac}} \approx 2$.

${ }^{3}$ Note that Run S04 is the same as Runs D01 and T01.
}

Table 1) show (from top to bottom) the vertical profiles of the mean toroidal velocity $\left(\bar{U}_{y}\right)$, the turbulent rms-velocity and $\bar{B}_{y}$ in the relaxed state. Through out this paper we consider averages taken first over the $y$ direction and then the averaging over the other directions is performed. Note that the $u_{\mathrm{rms}}$-profiles have been computed neglecting the mean flow. The curves of the turbulent velocity in the models that include the shear layer exhibit a bump where the shear is located. This feature does not appear in non-shearing models (see dotted line), suggesting that turbulent motions are developing in this regions. The bump is more pronunced in models with larger Sh which indicates that this turbulent motions are probably due to the $\mathrm{KH}$ instability. This difference in $u_{\text {rms }}$ translates into different values of the equipartition magnetic field, Eq. (13), which hence differs from model to model.

We find that the amplitude of the magnetic energy increases for deeper tachoclines. This is an expected result since below the convection zone the magnetic diffusivity has smaller values which allows storage of a larger magnetic flux. The vertical distribution of the energy of the mean toroidal magnetic field $\left(\bar{B}_{y}^{2}\right)$ peaks roughly at the center of the shear layer. The tail of this curve towards the convection zone may hint how buoyant the magnetic field is in each model. We expect that the larger the magnetic field (Run D04) the more magnetic flux becomes buoyant and rises into the upper layers.

The time evolution of the magnetic field of the runs in Set D are shown in the middle panel of Fig. 2. We find that the growth rate of the magnetic field, $\lambda=\mathrm{d} \ln B_{\mathrm{rms}} / \mathrm{d} t$, decreases slightly as the shear layer is moved deeper. This is not very clear in Table 1 since the error of this quantity is of the same order as the difference between different runs. However, it is clear from both the figure and the table, that the volume averaged magnetic field increases with the tachocline depth.

In the third group of simulations (Runs T01-T06), we increase the width of the shear layer gradually from $d_{\mathrm{s}}=0.05 d$ in Run T01 to $d_{\mathrm{s}}=0.20 d$ in Run T06. This change implies lower values of the shear parameter Sh, Eq. (12), but also a larger fraction of the tachocline in the turbulent and stable layers. The results, presented in Table 1 and depicted in Figs. 1 and 2 (see legends), indicate that smaller values of the shear, $\mathrm{Sh} \approx 3$, are still able to excite the dynamo at the price of a lower growth rate. For this set of simulations we find that thicker shear layers (i.e., smaller shear values) generate a larger fraction of mean magnetic field (e.g. Runs T03 and T04). This fact is a counter-intuitive result and does not agree with previous meanfield studies on influence of the thickness of the solar tachocline (Guerrero \& de Gouveia Dal Pino 2007). However, as it will be explained below, the important fact is that this configuration seems to be favorable to a longer storage of magnetic field in the 

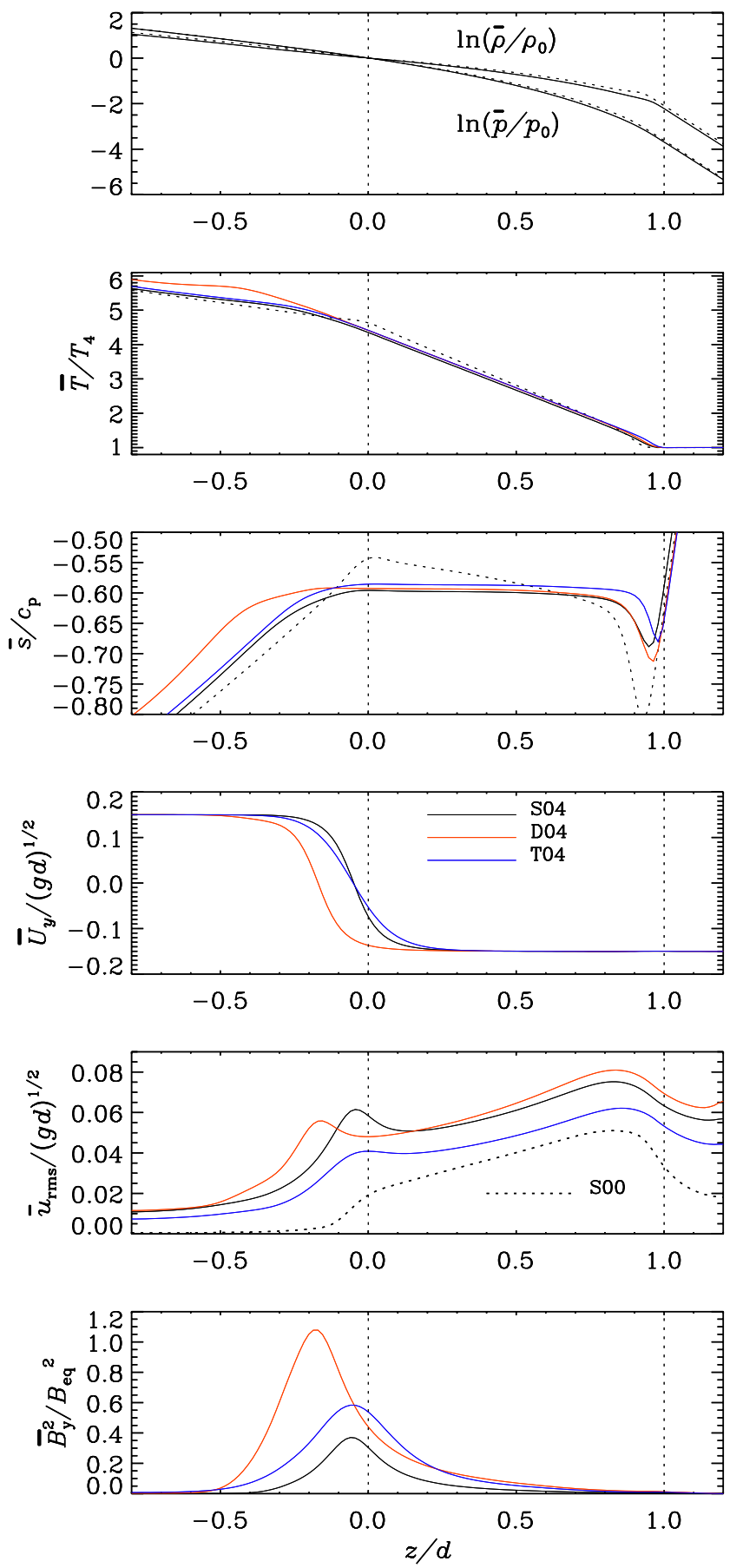

Fig. 1. Vertical profiles of mean density, pressure, temperature, specific entropy and azimuthal velocity $\left(\bar{U}_{y}\right)$ in the initial state (dotted lines) and in the thermally relaxed steady state (solid). The dotted vertical lines at $z=0$ and $z=d$ denote the bottom and top of the convectively unstable layer, respectively. The last two panels show the turbulent rms-velocity and the energy of the toroidal mean magnetic field in the saturated phase. For a better comparison, the rms-velocity profile of Run S00 has been multiplied by a factor of 2 .

stably stratified layer, with the advantage that here the effects of the shear on the thermal properties of the fluid are less important than in the previous sets of simulations. With the settings of Run T04 we find that the critical magnetic Reynolds number is between 8.1 (Run T04 $)$ and 4.1 (Run T04 ${ }_{3}$ ) as shown in Table 3. For the lower of the super-critical values of $\mathrm{Rm}$ (21.1 and 8.1), the magnetic field exhibits a long transient before it starts to


Fig. 2. Time evolution of the rms value of the total magnetic field normalized with the equipartition value at the center of the shear layer. Different lines/colors correspond to different runs as indicated in the legends in each panel.

grow. For this reason we have not ran these simulations until the field saturates. Run $\mathrm{T}_{0} 4_{3}$, on the contrary, $B_{\text {rms }}$ exhibits a clear exponential decay. We also notice that the growth rate depends on the magnetic Reynolds number.

Based on the results above, we perform another series of simulations whose parameters and results are summarized in Table 2. Runs AR01 and AR02 correspond to Run T04 but with aspect ratios $\left(L_{x}, L_{y}, L_{z}\right)=(8,4,2) d$ and $(4,8,2) d$, respectively. Runs D03b, and T04a to T04c have essentially the same 

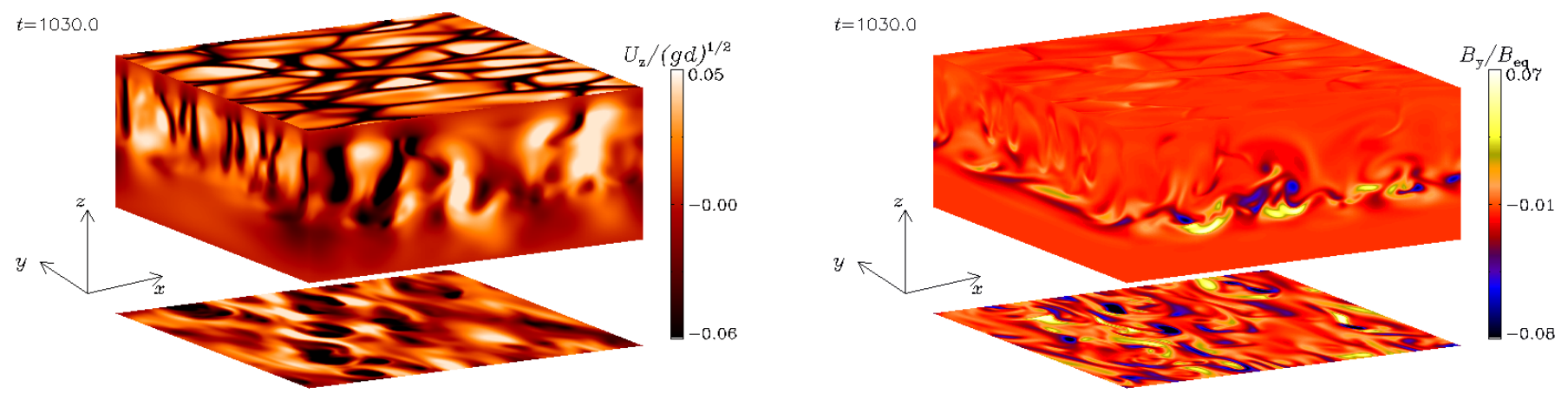

Fig. 3. Snapshots of the vertical velocity, $U_{z}$ (left) and the toroidal magnetic field, $B_{y}$ (right) in different planes of the domain, for Run T04c in the kinematic phase. The vertical slices correspond to the $y z$ (left side of the box) and $x z$ (front of the box) planes. The top plane corresponds to the horizontal boundary between the convective and the cooling layers at $z=d$ and the plane shown below the box corresponds to the base of the unstable layer $(z=0)$.

Table 3. Summary of runs with different magnetic Reynolds number.

\begin{tabular}{cccccc}
\hline \hline Run & $\mathrm{Sh}_{\mathrm{k}}$ & $\mathrm{Re}_{\mathrm{k}}$ & $\mathrm{Rm}_{\mathrm{k}}$ & $\mathrm{Ma}_{\mathrm{k}}$ & $\lambda\left[10^{-2}\right]$ \\
\hline $\mathrm{T} 04_{0}$ & 4.1 & 16.7 & 27.9 & 0.053 & 0.88 \\
$\mathrm{~T}_{1}$ & 4.1 & 16.8 & 21.1 & 0.052 & 0.72 \\
$\mathrm{~T}_{1} 4_{2}$ & 4.6 & 27.1 & 8.1 & 0.051 & 0.49 \\
$\mathrm{~T}_{4}$ & 4.5 & 27.7 & 4.1 & 0.052 & -0.39 \\
\hline
\end{tabular}

Notes. The parameters in these simulations correspond to those of Run T04 in Table 1 with different values of the magnetic diffusivity and viscosity. The quantities shown hare are defined in Table 1 but they are computed in the kinematic regime. Note that Run $\mathrm{T} 04_{0}$ corresponds to Run T04 in Table 1.

configuration than Runs D03 and T04 with $256^{3}$ grid points resolution. The values of $v$ and $K$ have been modified in order to obtain different Reynolds and Prandtl numbers.

The model with larger extent perpendicular to the direction of the shear $(x)$ does not show differences with respect to the reference case. On the other hand, the model with larger extent in the direction of the shear $(y)$ results in a reduced growth rate and in smaller values of the volume averaged magnetic field $\left(\tilde{B}_{\mathrm{rms}}\right)$, the maximum amplitude of the toroidal magnetic field and of the large-scale field. The reason for these differences is a change in the turbulent properties of the plasma for this elongated box. We notice that the rms velocity increases for this model (AR02) which reduces the effective value of the shear and other inductive effects. We searched for an explanation to these differences in purely hydrodynamic, isothermal, simulations of the $\mathrm{KH}$ instability (not reported here). We found that the turbulence induced by the $\mathrm{KH}$ instability gives rise to mean flows which differ, in amplitude and wave number, for different box aspect ratios. These modes may be responsible for affecting the dynamo properties. These interesting phenomena deserve a better clarification which we postpone to a forthcoming paper. Yousef et al. (2008a) have obtained that both the growth rate and the scale where the maximum magnetic energy is concentrated, converge to a value which is independent of the relevant length scale $\left(L_{z}\right.$ in their case). Such convergence analysis is computationally very expensive to be performed for our system.

The models with higher resolution (see Runs D03b and T04b in Table 2 and bottom panel of Fig. 2) show a larger growth rate when compared with their corresponding low resolution cases. These runs, however, saturate at sligthly smaller amplitudes of $\tilde{B}_{\text {rms. }}$. A similar weak dependence on Rm has been reported from simulations with horizontal shear (Käpylä et al. 2010a).
We have also changed $\mathrm{Rm}$ by considering a different input heat conductivity (i.e. different Pr). The results of Runs T04a-T04c show that $\lambda$ depends on Pr. There is no considerable difference between the rms magnetic field of these runs.

\subsection{Morphology of the dynamo-generated magnetic field}

In the initial stages of its evolution, the magnetic field is dominated by small scales and although it is possible to distinguish the stretching effects of the shear, the structures formed are small compared with the size of the box. In Fig. 3 we present snapshots of the Run T04c in the kinematic phase.

Mean magnetic fields take longer to develop in all simulations but in the saturated state they comprise a considerable fraction of the total field. This can be seen in the upper panel of Fig. 4 , where the ratio $b_{\text {rms }} / \bar{B}_{\text {rms }}$ for Run S04 is shown as a function of time. It is important to notice that mean values refer here to averages over the $y$ direction, i.e. $\bar{B}_{\mathrm{rms}}=\sqrt{\langle\boldsymbol{B}\rangle_{y}^{2}}$, and $b_{\mathrm{rms}}=\sqrt{\left\langle\boldsymbol{B}^{2}\right\rangle_{y}-\langle\boldsymbol{B}\rangle_{y}^{2}}$. We do not perform averaging over the $x$ direction because the toroidal magnetic field varies also in $x$ (see below). The average over $x$ and time is performed after the mean and fluctuation values are computed. In the same panel, the dashed line corresponds to the average value of this ratio $\left(b_{\mathrm{rms}} / \bar{B}_{\mathrm{rms}}=1.9\right)$ in the saturated phase of the dynamo, indicating that the mean magnetic field is roughly a third of the total magnetic field. For the sake of clarity only a single run is shown in this panel, but similar results are obtained for all simulations in Sets D and T.

In the bottom panel of Fig. 4, the vertical profiles of the mean (solid lines) and fluctuating (dashed lines) magnetic fields for four representative runs are shown. We find that the mean magnetic field is mainly located in the shear region, whereas the turbulent field, on the other hand, is more spread out inside the convection zone. It is noteworthy that for a thick tachocline the mean field is almost comparable with the fluctuating one, covering a fraction of the stable layer where the fluctuations are weak (see solid blue line). Simulations with higher resolution (see blue lines with diamond symbols in Fig. 4) exhibit fluctuating magnetic fields with vertical distribution and magnitude almost identical to the lower resolution case. The vertical profile of the mean field is roughly the same as in the lower resolution case but of smaller magnitude.

The structure of the magnetic field depends strongly on the structure of convection. In Fig. 5 we show snapshots of vertical 

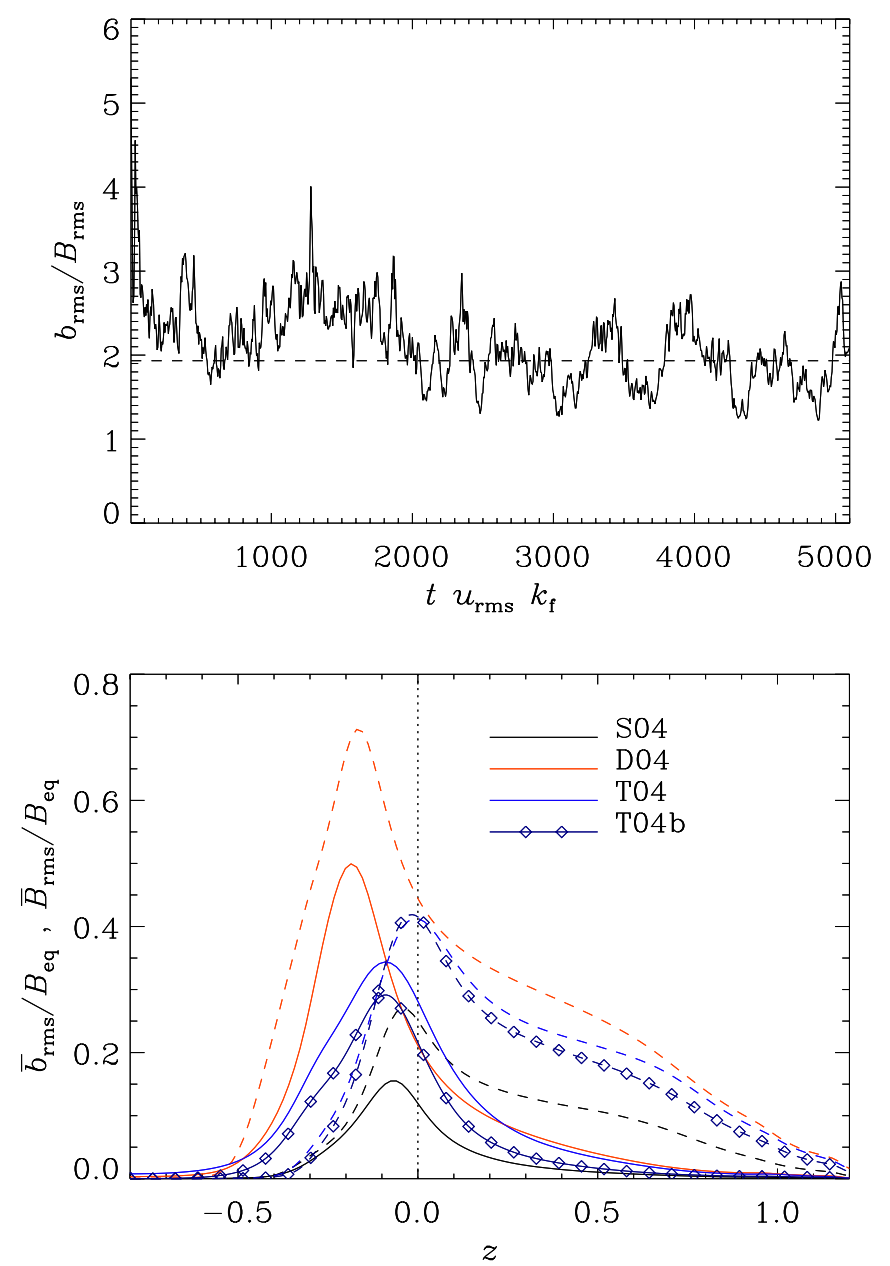

Fig. 4. Upper panel: ratio between the rms values of the small scale and mean magnetic fields, $b_{\mathrm{rms}}$ and $\bar{B}_{\mathrm{rms}}$, for Run S04. The dashed line corresponds to the mean value of this ratio in the saturated phase. Lower panel: radial distribution of the mean (dashed lines) and fluctuating (dashed lines) magnetic field for the Runs S04, D04, T04 and T04b in the saturated phase.

velocity $\left(U_{z}\right)$ and azimuthal magnetic field $\left(B_{y}\right)$ for arbitrary times in the saturated state for Runs S04, D04, and T04 (from top to bottom, respectively). From the right panels of this figure, it is possible to distinguish that at the base of the convection zone, the toroidal field organizes in elongated structures of both polarities which span all across the azimuthal direction if the penetrative (jet-like) downflows of the overshooting are less intense. These stripes coexist with more random magnetic fields in regions located where the downflows are able to reach the stable layer. The size of the magnetic structures is at least equal or larger than the scale of the convective eddies. This is more evident for the streamwise direction where the field occupies the full extent of the domain. The strong toroidal fields are mainly confined in the shear region. This can be seen in the right panels of Fig. 5 and also in the bottom panel of Fig. 1, where it is clear that the curve corresponding to Run T04 (blue line) has a broader profile than the one corresponding to Run S04 (black line). The presence of the $\mathrm{KH}$ instability is evident in the $y z$ plane where wave-like structures are observed. If the tachocline is thicker, the effects of the KH-instability are weaker. Another advantage of thicker shear layers is that they do not affect the thermodynamical state of the fluid as strongly as in Sets S and T. The disadvantage is that they produce broad and more diffuse magnetic fields which do not well agree with the flux tube scenario. This is discussed in more detail in the next section.

Although the resulting magnetic structures are buoyant (see the next section), we must clarify here that the $\alpha$ effect that sustains the magnetic field against diffusion here, in spite of Cline et al. (2003) does not depend, at least at the kinematic phase, on the buoyancy instability. It may result from the combination between the $\mathrm{KH}$ and convective instabilities. Our results are in contradiction with those of Tobias et al. (2008) who used a similar setup in the Boussinesq approximation. We believe that the lack of a large-scale dynamo in their simulations may be either due to the less step shear profile that they used or to the smaller value of the shear parameter. However, a visual inspection of their Fig. 11 suggests a large-scale dynamo with its mean field aligned in the $y$ direction.

\subsection{Buoyancy}

According to linear theory (Spruit \& van Ballegooijen 1982), thin magnetic flux tubes become buoyantly unstable when the condition $\beta \delta>-1 / \gamma$ is fulfilled. Here $\beta=2 \mu_{0} p / B^{2}$ is the plasma beta, $\delta=\nabla-\nabla_{\text {ad }}$ is the superadiabaticity, see Eq. (10), and $\gamma$ is the ratio of specific heats. This condition is satisfied everywhere in the convection zone where $\delta>0$. From this relation it follows that below the convection zone, where $\delta<0$, thin magnetic flux tubes require a field strength beyond a threshold value to become unstable. For a magnetic layer, the necessary and sufficient condition for the development of two-dimensional interchange modes, in which we are interested here, is given by Newcomb (1961):

$\frac{\mathrm{d} \rho}{\mathrm{d} z}>\frac{-\rho^{2} g}{\gamma p+B^{2} / \mu_{0}}$.

We find that for the cases with the thinnest shear layer, the instability region is above the center of the shear layer where the toroidal magnetic field reaches its maximum value (see Fig. 6). This implies that a large fraction of the magnetic field there becomes buoyantly unstable very quickly. For the cases with a thick shear layer (Set T), the magnetic field is unstable only within the convection zone. The magnetic field distribution of the Run T04 in Fig. 4 (blue line), indicates that a large fraction of the magnetic field is buoyantly stable. This configuration may explain why in these cases larger mean magnetic fields develop with smaller shear parameters than in Sets S and D.

Note, however, that this conclusion is based on averaged quantities. The evolution of local magnetic structures is rather complex and possibly also affected by the KH instability. The shear parameter in Run T04 is smaller compared to the Runs S04 and D04, so it is expected to have a less efficient $\mathrm{KH}$ instability and this should allow a longer stay of the magnetic field in the stable layer. This is hard to demonstrate since it is difficult to disentangle the effects of the different instabilities (convection, $\mathrm{KH}$, and buoyancy) in the simulations. Nevertheless, it seems that the longer the magnetic field stays in the stable region the greater the final mean magnetic field strength. Then, according to Fan (2001), the number of scale heights the magnetic flux concentrations may rise and its final structure depend only on how strong the field is in comparison to the turbulent convective motions.

In the simulations it is observed that when the magnetic field rises it expands as a consequence of decreasing density. The morphology of the magnetic fields in the $x z$ plane (see right panels of Fig. 5) is reminiscent of the mushroom-shape that has 
G. Guerrero and P. J. Käpylä: Dynamo action due to localized shear below a convection zone
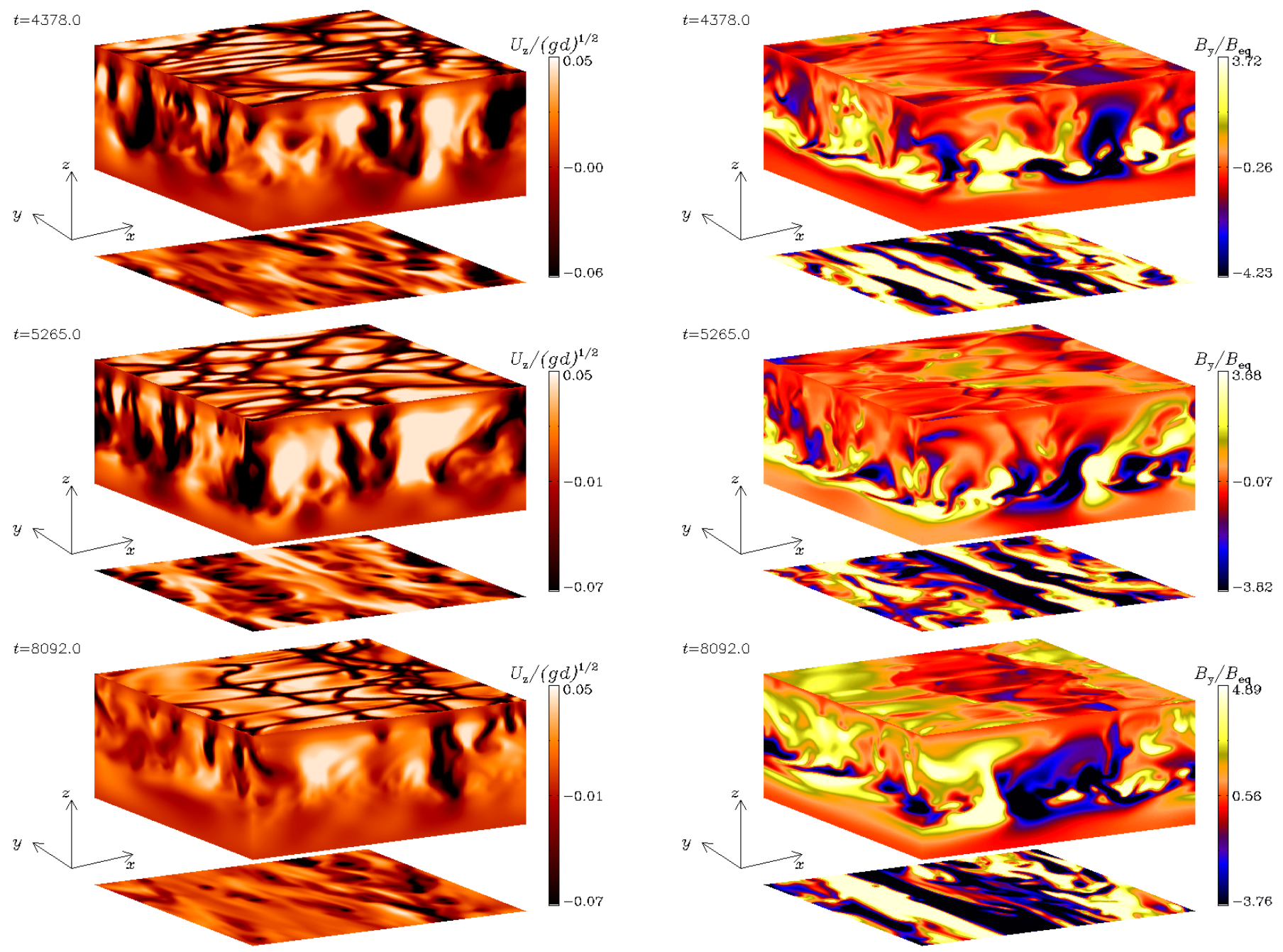

Fig. 5. Same as Fig. 3 but for Runs S04, D04 and T04 (from top to bottom) in the saturated phase. Movies corresponding to some of these runs may be found at: www. nordita.org/ guerrero.


Fig. 6. Buoyancy instability condition, Eq. (18), evaluated for Runs S04, D04 and T04. Continuous (dashed) lines correspond to the left (right) hand side of the equation, respectively. Dotted and dot-dashed lines correspond to the interfaces between convectively stable and unstable layers, and to the center of the tachocline in each simulation, respectively.

been obtained in several 2D (Schüssler 1979; Moreno-Insertis \& Emonet 1996; Longcope et al. 1996; Fan et al. 1998; Emonet \& Moreno-Insertis 1998) and 3D (Fan 2001; Fan et al. 2003; Fan 2008; Jouve \& Brun 2009) simulations of flux tube emergence. Such an expansion may result in the splitting and braking of the tube. We do not impose any twist on the tubes by hand since the dynamo generates the magnetic field self-consistently and the possibilities span from events that do not rise at all to events where buoyant magnetic fields rise up to the very surface. In the horizontal $y z$ plane we observe that the magnetic field lines 

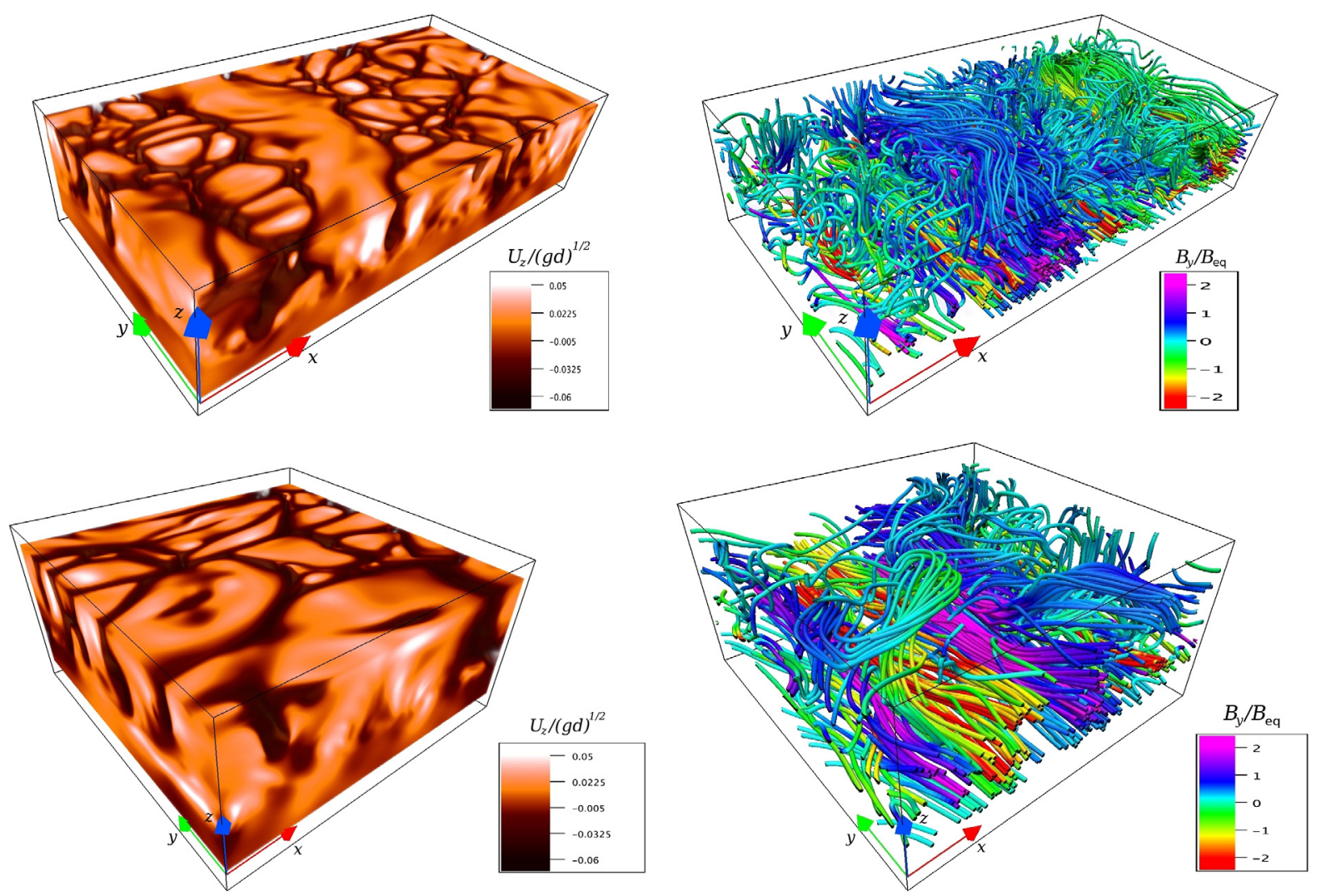

Fig. 7. Vertical velocity (left panels) and magnetic field lines (right panels) for emergence events in Runs AR01 (top) and T04c (bottom). The colors of the magnetic field correspond to the values of $B_{y} / B_{\text {eq }}$.

remain horizontal in the stable layer where they form. When the magnetic field rises, the field lines bend in the convection zone, with their rising part in the middle of upward flows and other parts withheld to the downward flows.

In Tables 1 and 2 we list the maximum values of $B_{y}$ in the simulations. In most of the cases we find $\max \left(B_{y}\right) \approx 5 B_{\text {eq }}$, and only in the models with a thicker (Runs T04 and AR01) or a deeper shear layer (Run D04), $\max \left(B_{y}\right)$ can be somewhat greater than $6 B_{\text {eq. }}$. Fan et al. (2003) argue that if $B_{0}>\left(H_{\mathrm{P}} / a\right)^{1 / 2} B_{\text {eq }}$, $B_{0}$ being the field strength in the shear layer and $H_{\mathrm{P}}$ the local pressure scale height, a buoyant flux-tube of radius $a$ may rise without experiencing the convective drag force. Here, although the shape of the magnetic field is not a tube, we compute the same condition using $a=d_{\mathrm{s}}$. We obtain values of $B_{0}$ going from $3.4 B_{\text {eq }}$ in Run D04 to $2.2 B_{\text {eq }}$ in Run T04 $\left(2.3 B_{\text {eq }}\right.$ in corresponding higher resolution runs). This indicates that the dynamo-generated magnetic field is supposedly insufficient to reach the surface without being modified by the convection. There are, however, a few cases in Runs D04, T04 and AR01, where the strongest magnetic fields are yet able to reach the surface. When this occurs the magnetic field reacts back on the flow and modifies the convective pattern. Broad convection cells elongated in the $y$ direction are formed. In the two upper panels of Fig. 7 we present one of these events for Run AR01. In the upper layers, the field lines show a turbulent pattern except in the center and the right edge of the box where the field lines at the surface cross the entire domain in the $y$ direction. In the higher resolution cases, we do not observe events where the field lines, in the uppermost layers, remain horizontal accross the whole toroidal direction. Nevertheless, the expanding magnetic field may reach the surface forming broad convection cells (at least two times larger than the regular cells). One of these cases, corresponding to Run T04c is shown in the bottom panels of Fig. 7. In this event two stripes of magnetic field of opposite polarity are reaching the surface simultaneously, the magnetic field lines of these ropes reconnect forming a large scale loop oriented in the $x$-direction.

In order to compute the rise speed of the magnetic field in these cases we have used the horizontal averages of $B_{y}$ and constructed the time-depth "butterfly" diagram shown in Fig. 8. The toroidal field is amplified in the shear layer, below $z=0$ (see dotted line). When it becomes buoyant it travels through the convection zone. The tilt observed in the contours of $B_{y}$ may give a rough estimate of the vertical velocity. In the same figure we have drawn correspondingly inclined white dashed lines to guide the eye. For the emergence events in Run D04 (AR01), the estimated rise speed is $u_{b} \approx 0.034(d g)^{1 / 2}\left(\approx 0.030(d g)^{1 / 2}\right)$. These values are small in comparison to the $u_{\text {rms }}$ values of the models (see Tables 1 and 2) which does not agree with the rise of a flux tube in a stratified atmosphere. For instance, Fan et al. (2003) obtain a final rise velocity $\approx 5$ times larger than the rms-velocity. We should point out that in the simulations here, the strong shear increases $u_{\text {rms }}$ by a factor of two or even more. If we compare the rise velocity of the magnetic field with the rms-velocity of a non-shearing case (Run S00), we find that $u_{b}$ is slightly larger. Magnetic pumping effects, which are evaluated below, may also play a role in braking the rise of magnetic structures and in determining its final velocity.

The results mentioned above do not fully agree with those found by Vasil \& Brummell (2008), where a toroidal magnetic 
G. Guerrero and P. J. Käpylä: Dynamo action due to localized shear below a convection zone

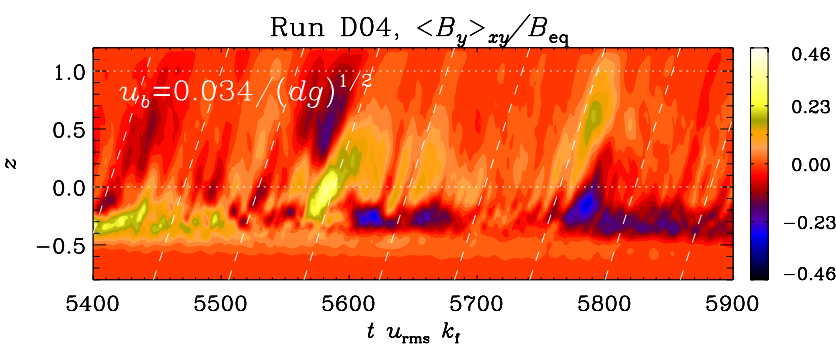

Run AR01, $\left\langle B_{y}>{ }_{x y} / B_{\text {eq }}\right.$



Fig. 8. Butterfly diagram in the depth-time plane for the horizontally averaged toroidal magnetic field, $B_{y}$ for Runs D04 and AR01. The dotted lines corresponds to the top $(z=d)$ and base $(z=0)$ of the convection zone. The tilted dashed lines show approximate trajectories of buoyant magnetic fields. A rough estimate of rise speed is computed from these lines as indicated. The event at $t u_{\mathrm{rms}} k_{\mathrm{f}}=9020$ in the bottom panel is also shown in the top panels of Fig. 7.

layer is generated from a purely vertical field through an imposed radial shear layer. Although their stratification is weaker than in our case, the buoyancy instability is hard to excite in their simulations and the buoyant rise events are slowed down rather quickly. The lack of a dynamo mechanism in their setup, able to sustain the magnetic field, is maybe the reason of its rapid diffusion.

\subsection{Back reaction}

Temporal analysis of helioseismic data has revealed that the solar angular velocity varies by around 5 per cent with respect to its mean value. The fluctuation pattern, like the sunspots, follows an 11-year cycle, which suggests that it corresponds to the back reaction of the magnetic field on the plasma motion (e.g. Basu \& Antia 2003; Howe et al. 2009). It is also known that the amplitude of the meridional circulation varies with the amplitude of the magnetic field, being smaller when the cycle reaches its maximum (see e.g. Basu \& Antia 2003). Apart from large scale effects, the magnetic field may also affect the local state of the plasma. This is possibly the case in the downflows found by Hindman et al. (2009); Zhao et al. (2010) nearby the bipolar active regions.

In the simulations presented here, where the dynamo presumably is different from that of the Sun, and due to the numerical setup, there are neither periodic oscillations of the magnetic field nor a large-scale circulation. We are able, however, to distinguish the differences between the plasma state in the kinematic and the saturated stages. We also follow the deviations of the averaged flow occurring during peaks of the magnetic field amplitude.

We find that the turbulent rms-velocity, as well as the imposed large-scale shear flow, are affected by the magnetic field. In the top panel of Fig. 9 we show the vertical profile of the horizontal and time averaged $u_{\mathrm{rms}}$ in the kinematic (dotted line) and saturated (solid line) regimes of the dynamo. A decrease in the amplitude is observed, especially in the region where


Fig. 9. Top: time averaged vertical profile of $u_{\mathrm{rms}}$ in the kinematic (dotted line) and saturated (solid line) stages for the Runs T04b. The dashed line shows the deviation from the average at a one of the peaks in the magnetic field amplitude. The three middle panels are butterfly diagrams of the angular velocity deviation, $\delta U_{y}=U_{y}-\bar{U}_{y}$, for the Runs AR01, T04b and T04 $\tau$. The continuous white lines in these panels indicate the time evolution of $B_{\mathrm{rms}}$ normalized to its maximum value in the time interval. The bottom panels show time-averaged (solid black lines) and instantaneous (dashed red lines, referring to the white dashdotted line in the bottom contour plot) values of $U_{y}$ and $\partial_{z} U_{y}$.

the magnetic field is more concentrated (dashed line). The volume averaged $u_{\text {rms }}$ also changes accordingly by $\sim 5$ percent, as indicated in Table 2. The dashed line in the top panel of Fig. 9 shows that it further decreases in the shear region.

In the middle panels of Fig. 9, butterfly diagrams of the toroidal velocity fluctuations, $\delta U_{y}=U_{y}-\bar{U}_{y}$, in the $z$-time 
plane are shown for the Runs AR01 and T04b, respectively. The dashed white line indicates the center of the shear layer. Positive values of this quantity below (above) the dashed line indicate faster (slower) toroidal velocities with respect to its mean value. Accordingly, negative values below (above) the dashed line indicate deficiency (excess) of the local $y$ flow. The continuous white line corresponds to the time evolution of $B_{\text {rms }}$ (for clarity normalized to its maximum value). We show this in order to demonstrate that the changes in $U_{y}$ depend on the amplitude of the magnetic field. The overall result of the contour plots is that during the maximum of the magnetic field the shear is slightly stronger. In these diagrams, as well as in all of the simulations listed in Tables 1 and 2 (except Run T04 $\tau$ ), the deviation from the averaged value of $U_{y}$ is found to be $\sim 3$ per cent.

The deviation of the mean shear profile depends on the forcing time scale $\tau_{\mathrm{f}}$ in Eq. (3). In Run T04 $\tau$, we consider the same settings as in Run T04, but varying $\tau_{\mathrm{f}}$ from $2(d / g)^{1 / 2}$ to $10(d / g)^{1 / 2}$ (i.e., $\approx 1$ to $\approx 5$ turnover times, respectively). The results show fluctuations of $\approx 10$ per cent of the shear velocity, and spread out to a wider fraction of the domain now (see bottom contour plot of Fig. 9). The change in the velocities is more clearly observed in the bottom panels of Fig. 9 where $\bar{U}_{y}$ is compared with $U_{y}$ at a time when the magnetic field peaks $\left(t u_{\mathrm{rms}} k_{\mathrm{f}}=4700\right.$ in Run $\mathrm{T} 04_{\tau}$, see the dot-dashed line in the corresponding butterfly diagram). While the profiles of the velocities are slightly different, the instantaneous shear is clearly larger than the time-averaged one. Since magnetic buoyancy is present also at the time of the maximum, the toroidal velocity in the middle of the convection zone is also affected by the rising flux. This change is not so strong as the one in the shear layer but is also a general characteristic of the simulations. Although this is a counterintuitive result, we have verified the same effect in all the simulations during maxima of the magnetic field strength. Disentangling the contribution of the Lorentz force that leads to this change in the $y$ velocity is not simple. We conjecture that the positive contribution to the shear depends mostly on the term $B_{x} J_{z}$ which dominates the $y$-component of the Lorentz force.

Similar results have been found in global numerical simulations of oscillating dynamos by Brown et al. (2011). They found torsional fluctuations in the surface angular velocity at higher latitudes (see their Fig. 11). These oscillations seem to follow the poleward migration of strong toroidal magnetic field (see the bottom panel of their Fig. 1). Correspondingly, surface fluctuations of the solar angular velocity exhibit an equatorial branch which seems to follow the contours of the strongest magnetic field. The results of the simulations presented here and those of Brown et al. (2011) are suggestive of the magnetic nature of this branch in the solar torsional oscillation pattern. Besides, a highly super-equipartition magnetic field stored at the tachocline should produce a significant change in the radial shear there. Such changes, if present, should possibly be detectable by helioseismology in forthcoming years.

\subsection{Turbulent transport coefficients}

In order to study the underlying mechanism of the mean-field dynamo in our simulations, we compute the turbulent transport coefficients from a representative simulation (Run T04). In order to do this, we use the test-field method, introduced in the geodynamo context by Schrinner et al. $(2005,2007)$ and currently available in the Pencil Code (e.g. Brandenburg 2005b; Brandenburg et al. 2008).
In mean-field theory, the electromotive force $\overline{\mathcal{E}}=\overline{\boldsymbol{u} \times \boldsymbol{B}}$ governs the evolution of the large-scale magnetic field (Krause \& Rädler 1980). We assume that there is scale separation between the mean field and the fluctuating flow. Considering large-scale fields that depend only on $z$, the electromotive force may be written in terms of the large-scale magnetic field:

$\overline{\mathcal{E}}_{i}=\alpha_{i j} \bar{B}_{j}-\eta_{i j} \bar{J}_{j}$

Four independent test fields are needed in order to compute the $4+4$ components of $\alpha_{i j}$ and $\eta_{i j}$ (see a detailed description of the method in Brandenburg et al. 2008). Even though we are aware of the possible dependency of the turbulent coefficient on the scale of the mean-field (see e.g. Chatterjee et al. 2011), we restrict ourselves for simplicity here to linear test fields only. The novel feature of the test-field method is that the test fields do not act back on the flow and that the turbulent diffusivity can also be computed, thus avoiding many of the problems that plague other methods (cf. Käpylä et al. 2010b).

We discuss our results in terms of the following quantities:

$\alpha_{x x}=\alpha_{11}, \quad \alpha_{y y}=\alpha_{22}$,

$\eta_{t}=\frac{1}{2}\left(\eta_{11}+\eta_{22}\right), \quad \epsilon_{\eta}=\frac{1}{2}\left(\eta_{11}-\eta_{22}\right)$,

$\gamma=\frac{1}{2}\left(\alpha_{21}-\alpha_{12}\right), \quad \epsilon_{\gamma}=\frac{1}{2}\left(\alpha_{21}+\alpha_{12}\right)$,

which represent the inductive $(\alpha)$, diffusive $\left(\eta_{\mathrm{t}}\right)$, and pumping $(\gamma)$ effects of turbulence, respectively. Furthermore, $\epsilon_{\eta}$ describes the anisotropy of the turbulent diffusion, and $\epsilon_{\gamma}$ is the symmetric part of the off-diagonal components of $\alpha$. The latter can be related to field-direction dependent pumping (cf. Ossendrijver et al. 2002). In Fig. 10 we show the vertical profiles of the kinetic helicity, $\overline{\boldsymbol{u} \cdot \boldsymbol{\omega}}$, where $\omega=\boldsymbol{\nabla} \times \boldsymbol{u}$, is the vorticity, and the turbulent transport coefficients of Eqs. (20)-(22), normalized with the first order smoothing approximation (FOSA) quantities:

$\alpha_{0}=\frac{1}{3} u_{\mathrm{rms}}, \quad \eta_{t 0}=\frac{1}{3} u_{\mathrm{rms}} k_{\mathrm{f}}^{-1}$.

For computing the coefficients in Fig. 10 we use Run T04 in the purely hydrodynamic state. The magnetic Reynolds number in the test field run is $\mathrm{Rm} \approx 16$. This value is above the critical $\mathrm{Rm}$ for dynamo excitation. Previous studies have shown that the turbulent transport coefficients for convection are independent of the magnetic Reynolds number provided that Rm is sufficiently larger than unity (Käpylä et al. 2009a). Thus the results of this run can be considered representative of the present setup.

We find that a certain amount of helicity is generated in the system, but that it is still statistically consistent with zero. The coefficient $\alpha_{x x}$ shows large fluctuations around zero. This component of $\alpha$ contributes to amplifying the generation of $B_{y}$ out of $B_{x}$, but its contribution is likely to be negligible when compared with that of the shear in the present models.

The component $\alpha_{y y}$ shows a zero mean value, but its variance has a large amplitude, especially in the middle of the convection zone. According to Vishniac \& Brandenburg (1997), such an incoherent $\alpha$ effect, being zero in average but having finite variance, may generate enough inductive effects to sustain the dynamo. Hence, this effect may be is the mechanism sustaining the dynamo here.

The shear-current effect, due to the mean shear flow interacting with turbulence, may also result in the generation of a mean field (Rogachevskii \& Kleeorin 2003, 2004). The existence of this effect has been studied with models of forced turbulence and latitudinal shear by (Brandenburg et al. 2008; Mitra et al. 2009), but no conclusive evidence has been found. Similar results have 
G. Guerrero and P. J. Käpylä: Dynamo action due to localized shear below a convection zone
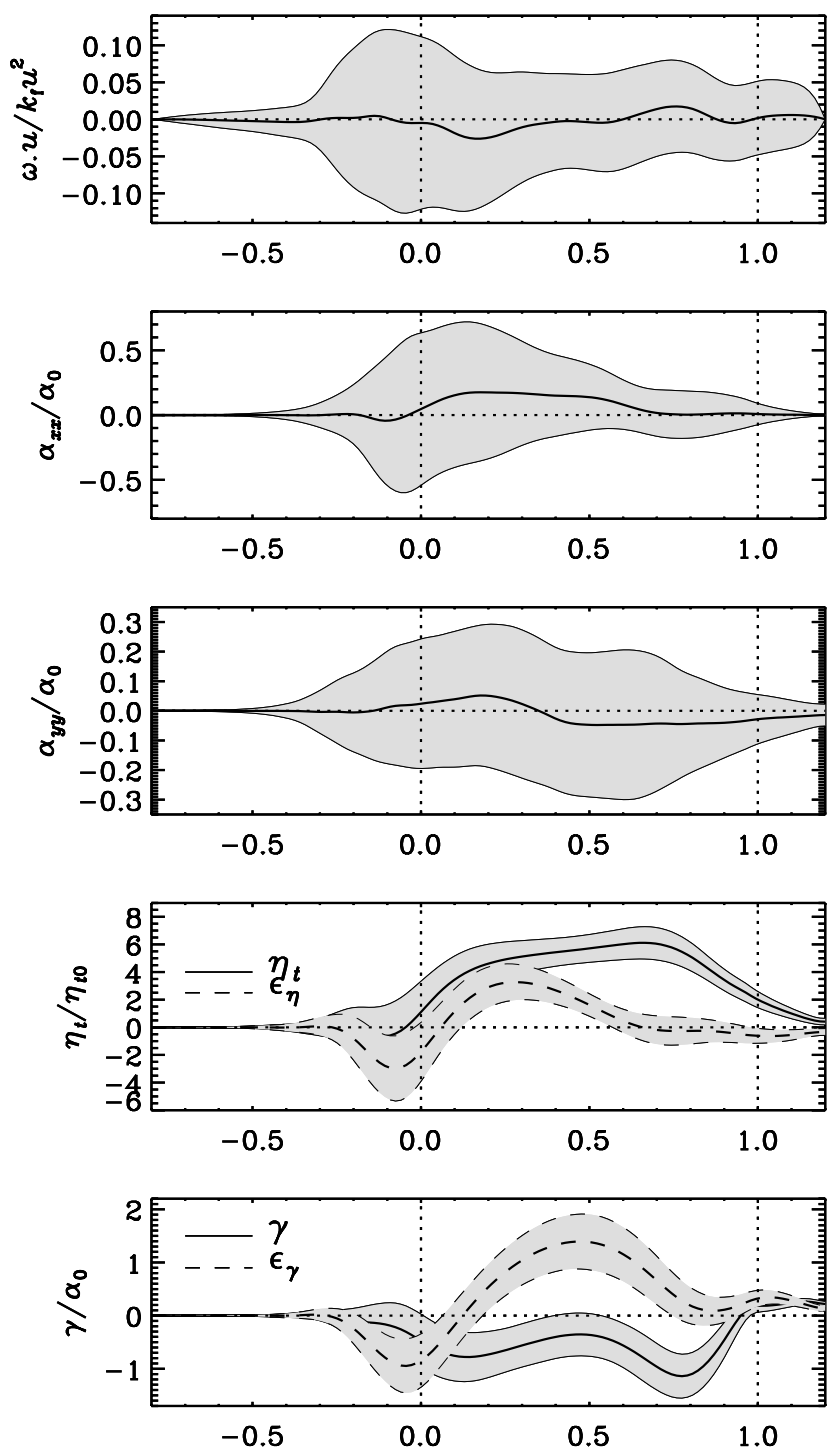

Fig. 10. Normalized profiles of the kinetic helicity, and the turbulent transport coefficients $\alpha_{x x}, \alpha_{y y}, \eta_{\mathrm{t}}, \epsilon_{\eta}, \gamma$ and $\epsilon_{\gamma}$ (from top to bottom) computed with the test-field method.

also been found from convection with horizontal shear (Käpylä et al. 2009a). In the case with vertical shear presented here, the relevant coefficient for this effect is not captured by the current version of the test-field method with only $z$-dependent test fields. Thus, we leave the study of the shear-current effect in turbulent convection with vertical shear for a forthcoming work.

The turbulent diffusivity, $\eta_{\mathrm{t}}$, is roughly six times larger than the FOSA estimate. This result agrees with previously computed turbulent diffusivity values for convection (Käpylä et al. 2009a). The vertical profile of $\eta_{\mathrm{t}}$ differs somewhat from these results, indicating the effect of the radial shear on the turbulent diffusion. Another interesting point is that $\epsilon_{\eta}$ is not close to zero, as in Käpylä et al. (2009a). This is likely explainable by the stronger shear used here. It is also an indication of the tensorial character of $\eta_{\mathrm{t}}$ and suggests that the distinct components of the magnetic field may diffuse differently.

Finally, the vertical profile of the turbulent pumping $(\gamma)$ in Fig. 10 shows that there is downwards transport of the mean magnetic field almost everywhere in the convection zone and upward transport only close to the top. The pumping velocity is

comparable with $u_{\mathrm{rms}}$ but it is not sufficient to retain the magnetic field within the stable layer, which indicates that buoyancy is a more efficient mechanism than magnetic pumping in transporting magnetic fields. The downward pumping is often attributed to the diamagnetic effect. Note, however, that the amplitude of this effect depends on the gradient of the level of turbulence, $\gamma_{\text {dia }}=-\nabla \eta_{\mathrm{t}} / 2$ (Kichatinov \& Rüdiger 1992), which is not very large in the current simulations. However, other effects, such as non-locality can affect the pumping velocity (Käpylä et al. 2009a).

\section{Conclusions}

We perform numerical simulations of turbulent convection with a thin vertical shear layer with the aim of modeling some of the relevant physics of the solar convection zone and the tachocline self-consistenly. Our model includes strong shear at the base of the convection zone and magnetic buoyancy. However, our model omits the overall rotation of the star which is very likely to be important in driving the dynamo in the Sun. The shear layer is located below the interface between a convectively stable and unstable layers. We consider the velocity gradient, the position in depth and the thickness of this layer as free parameters of the model and concentrated on two phenomena relevant for the solar cycle: dynamo process and magnetic buoyancy.

We find that it is possible to excite a large scale dynamo by the combined action of turbulent convection and a localized radial shear. The efficiency of the dynamo (i.e., its growth rate), and the maximum amplitude of the magnetic field depend on the shear amplitude, the thickness of the shear layer, the aspect ratio and the magnetic Reynolds number of the system (see Fig. 2 and Tables 1 and 2).

The magnetic field is organized in elongated structures in the direction of the shear at the base, or below, of the convection zone. The large-scale magnetic field can have either polarity and its strength may reach up to 40 percent of the total field (solid lines in Fig. 4). It coexists with a turbulent magnetic field which is distributed all across the convection zone. In the stable layer the fluctuating magnetic field exists in the regions of strong downflows. For fixed depth and thickness (Set $\mathrm{S}$ ) a larger growth rate and higher saturated $B_{\mathrm{rms}}$ is obtained for a larger shear parameter. A critical value of $\mathrm{Sh} \approx 7$ is required to excite the dynamo.

If the tachocline is located deeper (simulations in set D), the magnetic field develops mainly in the convectively stable (but still to some extent turbulent) layer which allows a longer field storage. In these cases the dynamo grows faster and with a larger fraction of mean magnetic field. This configuration, however, is not optimal since with the numerical resolution used here the thermodynamical state of the fluid is affected by viscous heating in the shear region. If the depth of the shear layer remains constant but the thickness increases (Set T), the magnetic field grows more slowly but it contains a larger fraction of mean magnetic field. This may be a consequence of the smoother shear profile which renders the magnetic field buoyantly unstable only in the superadiabatic part of the domain (see right panel of Fig. 6). A lower critical shear number is found to be able to excite the dynamo instability in these cases $(\mathrm{Sh} \approx 3)$.

Larger magnetic Reynolds numbers are achieved in two ways, firstly by doubling the resolution and secondly by changing the heat conductivity (i.e., changing Pr). On one side, for a fixed Pr and larger Rm, the dynamo exhibits a much faster growth. On the other hand, for a fixed resolution and different heat flux, the growth rate changes proportionally to Pr. In these 
higher resolution runs the final value of $B_{\text {rms }}$ is slightly below that in the corresponding cases with lower resolution. This may correspond to the dependence of the saturation value of the magnetic field on the magnetic Reynolds number or may also be the result of insufficient statistics.

Since the system is not rotating, there is no kinetic helicity and hence no $\alpha$-effect. The test-field analysis suggests that the probable mechanism responsible for the amplification of the magnetic field could be the incoherent $\alpha$-shear dynamo. However, other possibilities like the shear-current effect can not be ruled out for the time being.

Magnetic buoyancy is observed in all of the simulations. Based on horizontal averages we analyze the instability condition for 2D interchange modes (Eq. (18) and Fig. 6). We find that in models with a deeper tachocline the buoyancy instability develops even in the stable layer whereas in models with thicker shear layers the magnetic field is unstable in the convection zone only. This, however, does not mean that there are no emergence events. As far as the toroidal magnetic field is strong enough it rises through the convection zone forming mushroom-shaped structures.

When the buoyant magnetic field is weak it is strongly modified by local convective flows (see Fig. 5). On the other hand, magnetic fields of strong amplitude formed in the shear layer are able to rise up to the surface and modify the convective pattern (Fig. 7). They may form either large convection cells or convective rolls that occupy the full $y$-extent. The clearest events are observed when the magnetic field in the shear layer exceeds the equipartition field by a factor $\geq 6$. Such strong magnetic fields have been observed only in simulations with $128^{3}$ grid points resolution. The rise velocity of the buoyant field in the bulk of the convection zone, estimated from depth-time "butterfly" diagrams, has been found to be $\approx 0.6 u_{\mathrm{rms}}$. From the test-field method results, the maximal downward pumping velocity of magnetic field is found to be $\gamma \approx 0.4 u_{\mathrm{rms}}$. This indicates that buoyancy is indeed efficient in transporting magnetic flux from the base of the convection zone. However, the magnetic field structures expand very quickly during the rise and magnetic structures observed close to the upper boundary are no longer so well organized.

Besides the emergence of the magnetic field that affects the flow pattern locally, other changes are observed due to the backreaction of the magnetic field on the plasma. In the saturated phase, the rms-velocity changes from its kinematic value to a lower amplitude when the magnetic field is strong. In addition, during the peaks and valleys of the field amplitude, the imposed shear profile presents systematic variations with respect to its averaged profile. For most of the models this change is around three percent of the shear velocity and is reminiscent of the fluctuations observed in the solar rotation profile or "torsional oscillations". The variation signal is mainly observed in the places where $\boldsymbol{B}$ is strong, with much weaker changes within the convection zone. These results encourage the study of the torsional oscillations in detail through direct numerical simulations.

Acknowledgements. We are thankful to the referee and Matthias Rheinhardt for their valuable comments on this work. The authors acknowledge the hospitality of NORDITA. This work is supported by the European Research Council under the AstroDyn research project 227952. The computations were performed under the HPC-EUROPA2 project (project number: 228398) with the support of the European Commission - Capacities Area - Research Infrastructures. Field line tracing figures were produced using VAPOR. P.J.K. acknowledges the financial support from the Academy of Finland grant Nos. 121431, 136189, and 140970.

\section{References}

Abbett, W. P., Fisher, G. H., \& Fan, Y. 2001, ApJ, 546, 1194

Basu, S., \& Antia, H. M. 2003, ApJ, 585, 553

Brandenburg, A. 2005a, ApJ, 625, 539

Brandenburg, A. 2005b, Astron. Nachr., 326, 787

Brandenburg, A., \& Subramanian, K. 2005, Phys. Rep., 417, 1

Brandenburg, A., Jennings, R. L., Nordlund, Å., et al. 1996, J. Fluid Mech., 306, 325

Brandenburg, A., Rädler, K., Rheinhardt, M., \& Käpylä, P. J. 2008, ApJ, 676, 740

Brandenburg, A., Kemel, K., Kleeorin, N., \& Rogachevskii, I. 2010a [arXiv: 1005.5700 ]

Brandenburg, A., Kleeorin, N., \& Rogachevskii, I. 2010b, Astron. Nachr., 331, 5

Brown, B. P., Miesch, M. S., Browning, M. K., Brun, A. S., \& Toomre, J. 2011, ApJ, 731, 69

Caligari, P., Moreno-Insertis, F., \& Schussler, M. 1995, ApJ, 441, 886

Chatterjee, P., Mitra, D., Rheinhardt, M., \& Brandenburg, A. 2011, A\&A, accepted [arXiv: 1011.1218$]$

Cheung, M. C. M., Schüssler, M., Tarbell, T. D., \& Title, A. M. 2008, ApJ, 687, 1373

Christensen-Dalsgaard, J., \& Thompson, M. J. 2007, in The Solar Tachocline, ed. D. W. Hughes, R. Rosner, \& N. O. Weiss, 53

Cline, K. S., Brummell, N. H., \& Cattaneo, F. 2003, ApJ, 599, 1449

Emonet, T., \& Moreno-Insertis, F. 1998, ApJ, 492, 804

Fan, Y. 2001, ApJ, 546, 509

Fan, Y. 2008, ApJ, 676, 680

Fan, Y., \& Fisher, G. H. 1996, Sol. Phys., 166, 17

Fan, Y., Zweibel, E. G., \& Lantz, S. R. 1998, ApJ, 493, 480

Fan, Y., Abbett, W. P., \& Fisher, G. H. 2003, ApJ, 582, 1206

Guerrero, G., \& de Gouveia Dal Pino, E. M. 2007, A\&A, 464, 341

Hindman, B. W., Haber, D. A., \& Toomre, J. 2009, ApJ, 698, 1749

Howe, R., Christensen-Dalsgaard, J., Hill, F., et al. 2009, ApJ, 701, L87

Jouve, L., \& Brun, A. S. 2009, ApJ, 701, 1300

Käpylä, P. J., \& Brandenburg, A. 2009, ApJ, 699, 1059

Käpylä, P. J., Korpi, M. J., \& Brandenburg, A. 2008, A\&A, 491, 353

Käpylä, P. J., Korpi, M. J., \& Brandenburg, A. 2009a, A\&A, 500, 633

Käpylä, P. J., Korpi, M. J., \& Brandenburg, A. 2009b, ApJ, 697, 1153

Käpylä, P. J., Korpi, M. J., \& Brandenburg, A. 2010a, A\&A, 518, A22

Käpylä, P. J., Korpi, M. J., \& Brandenburg, A. 2010b, MNRAS, 402, 1458

Käpylä, P. J., Brandenburg, A., Kleeorin, N., Mantere, M. J., \& Rogachevskii, I.

2011, MNRAS, submitted [arXiv: 1104.4541]

Kichatinov, L. L., \& Rüdiger, G. 1992, A\&A, 260, 494

Kleeorin, N., \& Rogachevskii, I. 1994, Phys. Rev. E, 50, 493

Krause, F., \& Rädler, K. 1980, Mean-field magnetohydrodynamics and dynamo theory (Oxford: Pergamon Press), 1980

Longcope, D. W., Fisher, G. H., \& Arendt, S. 1996, ApJ, 464, 999

Martínez-Sykora, J., Hansteen, V., \& Carlsson, M. 2008, ApJ, 679, 871

Mitra, D., Käpylä, P. J., Tavakol, R., \& Brandenburg, A. 2009, A\&A, 495, 1

Moreno-Insertis, F., \& Emonet, T. 1996, ApJ, 472, L53

Newcomb, W. A. 1961, Phys. Fluids, 4, 391

Ossendrijver, M., Stix, M., Brandenburg, A., \& Rüdiger, G. 2002, A\&A, 394, 735

Parker, E. N. 1955, ApJ, 121, 491

Rogachevskii, I., \& Kleeorin, N. 2003, Phys. Rev. E, 68, 036301

Rogachevskii, I., \& Kleeorin, N. 2004, Phys. Rev. E, 70, 046310

Rogachevskii, I., \& Kleeorin, N. 2007, Phys. Rev. E, 76, 056307

Schrinner, M., Rädler, K., Schmitt, D., Rheinhardt, M., \& Christensen, U. 2005, Astron. Nachr., 326, 245

Schrinner, M., Rädler, K., Schmitt, D., Rheinhardt, M., \& Christensen, U. R. 2007, Geophys. Astrophys. Fluid Dynam., 101, 81

Schüssler, M. 1979, A\&A, 71, 79

Silvers, L. J., Bushby, P. J., \& Proctor, M. R. E. 2009a, MNRAS, 400, 337

Silvers, L. J., Vasil, G. M., Brummell, N. H., \& Proctor, M. R. E. 2009b, ApJ, 702, L14

Spruit, H. C. 1981, A\&A, 98, 155

Spruit, H. C., \& van Ballegooijen, A. A. 1982, A\&A, 106, 58

Tobias, S. M., Brummell, N. H., Clune, T. L., \& Toomre, J. 1998, ApJ, 502, L177

Tobias, S. M., Brummell, N. H., Clune, T. L., \& Toomre, J. 2001, ApJ, 549, 1183

Tobias, S. M., Cattaneo, F., \& Brummell, N. H. 2008, ApJ, 685, 596

Vasil, G. M., \& Brummell, N. H. 2008, ApJ, 686, 709

Vishniac, E. T., \& Brandenburg, A. 1997, ApJ, 475, 263

Yousef, T. A., Heinemann, T., Rincon, F., et al. 2008a, Astron. Nachr., 329, 737

Yousef, T. A., Heinemann, T., Schekochihin, A. A., et al. 2008b, Phys. Rev. Lett., 100,184501

Zhao, J., Kosovichev, A. G., \& Sekii, T. 2010, ApJ, 708, 304 